Comportamientos

Judiciales Impropios

a través de redes

sociales

Pamela Güemes Vassallo 


\section{Comportamientos Judiciales Impropios a través de redes sociales.}

Improper Judicial Behaviors
through social networks

Pamela Güemes Vassallo

Abogada, egresada de la Universidad Nacional de Córdoba (Argentina). https://orcid.org/0000-0003-4294-1274

Pamela pame_g4@hotmail.com

Recibido: 16 enero 2020

Aceptado: 10 marzo 2020

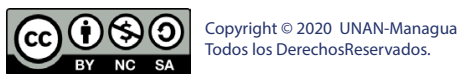

\section{RESUMEN}

En las líneas siguientes se analiza el caso del magistrado Colón Colón de Puerto Rico a través del concepto de Comportamientos Judiciales Impropios, tras las publicaciones que realizó en su página de Facebook en el año 2017. La exposición de dicho caso no es el asunto central del trabajo sino que es una especie de disparador que se utiliza a exponer una serie de interrogantes que surgen a la hora de considerar aspectos vinculados a cuestiones morales (más que técnicas) en una decisión judicial. Puede observarse que a lo largo del ensayo son más las dudas que las respuestas concretas que se encuentran acerca de algo, debido a que, el objetivo central es reflexionar acerca de si los magistrados judiciales deben tener determinadas cualidades morales, $y$, a partir de ello, en el caso de que se optase por una respuesta afirmativa, buscar una justificación acerca de porqué deben ser de una forma u otra.

\section{ABSTRACT}

In the following lines is analised the Colón Colón magistered case from Puerto Rico, using the concept of "Inappropiate Magisterial Behaviour" since the publications He made on his Facebook page in the year 2017. The exposition of this case is not the central part of the essay but it is the base that we are going to use to point a series of questions. Those questions come up in order to considerate aspects vinculated to moral questions (no technical) in a judicial decision. As the essay continues, we can observe that there are more questions than actual answers, since the hole purpose is to debate whether the judicial magistered should have moral qualities, and justify that answer.

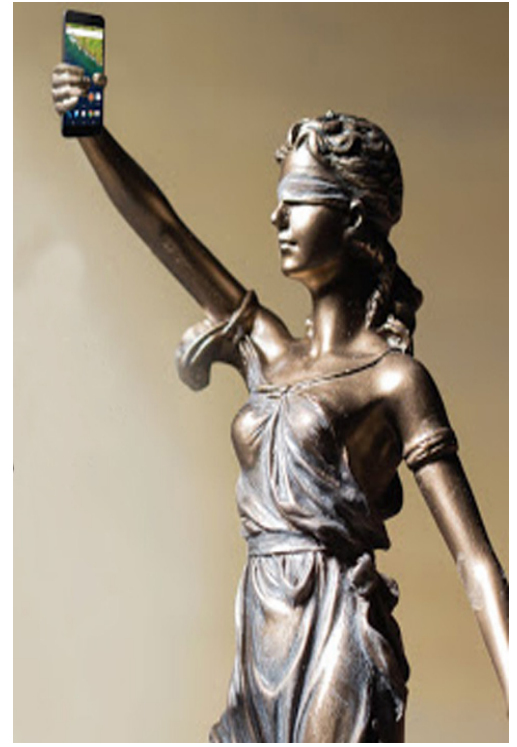

\section{PALABRAS CLAVE}

Comportamientos Judiciales Impropios, Redes Sociales, Virtud,Ética Judicial.

\section{KEYWORDS}

Improper Judicial Behaviors, Social Networks, Virtue, Judicial Ethics 


\section{Introducción}

En el presente trabajo se analizarán los llamados comportamientos impropios de los jueces (en adelante "CJI") cometidos a partir del uso de redes sociales. Se tendrán en cuenta las conductas públicas y las privadas con trascendencia pública realizadas de manera indebida por parte de los magistrados.

Será puesto a consideración el caso del juez Eric Colón Colón del municipio de Coamo en Puerto Rico, quien fue suspendido de su cargo tras realizar una serie de publicaciones indebidas vía Facebook. El estudio de dichos comportamientos será abordado a partir de criterios éticos judiciales y utilizando principalmente el concepto de CJI como así también la teoría de la virtud aristotélica.

Por otro lado, se expondrán algunos aspectos de carácter genérico desde los cuales se parte para llevar a cabo el análisis de la temática propuesta y se hará referencia a los Códigos de Ética. Además, se hará mención al Principio de Posibilidades Alternativas (en adelante "PPA") a los fines de evaluar si ante la realización de un comportamiento impropio resulta posible otro curso de acción y como consecuencia de ello la formulación de un reproche.

Finalmente, se expondrán algunas conclusiones las cuales se caracterizan por presentar más interrogantes que respuestas. Se trata de una temática en la que es necesario continuar pensando determinadas cuestiones a los fines de lograr una sociedad más pacífica, donde la mayoría de los ciudadanos posean una mejor calidad de vida, y, ello guarda relación la calidad de magistrados que posea una sociedad.

\section{El caso (los hechos):}

Con fecha 31/03/2017 el juez Eric Colón Colón, magistrado de Primera Instancia del municipio de Coamo en Puerto Rico, fue suspendido tres meses por el Tribunal Supremo de dicho país atento haber realizado publicaciones impropias en su Facebook.

Se transcriben algunos de los comentarios desplegados por el juez plasmados en el desarrollo del fallo del Tribunal:

a) "Una señora me dice: 'no he podido pagar la renta porque a mi marido le dieron lay oss'. Y yo en mi mente: Ay chuss!"; b) "Que bonita esta querella que me han traído (sic)!". Junto al comentario el juez Colon Colon publico dos fotografias que parecen ser porciones de una querella manuscrita; c) "Esta señora me ha dicho hoy que su hijo padece de esquizofrenia, pero anenoide. Diiitoooo"; d) "Sigo acordándome de cosas: Hace algún tiempo un señor se excuso porque no pudo complacer al tribunal. Yo le conteste que no habia ningún problema, que el tribunal estaba de lo mas bien, pero que gracias por preocuparse. Lolll”; e) "Asi (sic) mismo como lo lee: esta persona presentó una querella porque le vendieron un carro sin batería y sin valvete"; f) "Entonces, la peticionaria de la orden de protección, al llenar el encasillado donde se describe el tipo de relación que sostenía con el peticionado, esto fue lo que escribió" Junto al comentario, aparece una fotografía que parece ser un formulario de orden de protección de la OAT. En el encasillado del formulario donde se indicaba "otra", la persona escribió 'me endrogue (sic)’.1. 
El fundamento utilizado por el Tribunal a los fines de la suspensión fue que en el caso en cuestión el magistrado infringió los Cánones de la Ética Judicial, considerando que a los fines de la función judicial no alcanza solamente con la correcta aplicación de la norma sino que se requiere también un determinado comportamiento por parte de los jueces².

\section{Comportamientos judiciales impropios:}

En cuanto a la conceptualización de los CJI se parte de lo manifestado por el Dr. Armando Andruet (h): “(...) bajo el concepto de CJI percibimos un universo de situaciones que, si bien pueden ser cumplidas en el ámbito público de la labor judicial, por defecto se materializan en comportamientos efectuados en el ámbito privado del juez pero que tienen ellos trascendencia pública.

Además de lo público y lo privado con trascendencia pública, existen los comportamientos intimos o privadísimos del juez, que quedan fuera de cualquier ponderación posible de CJI. Ello así, porque en principio se tratarían de conductas sólo autorreferentes del nombrado, aunque hay que señalar también que no es del todo pacifica dicha conclusión y hacemos nuestra dicha crítica bajo ciertas circunstancias, pero eso no está en debate ahora.

Los CJI públicos son aquellos que en términos generales afectan o parecen afectar las prácticas virtuosas de los jueces, en cuanto tal conducta se produce mientras el juez está ejercitando biográficamente un cumplimiento activo del rol social que la judicatura le impone. Esto es: en todas aquellas circunstancias temporales o materiales en las cuales sólo se explica su participación por el mismo ejercicio del rol público institucional que tiene (...)”’.

Es decir, se entiende por dichos comportamientos aquellos que son llevados a cabo por los magistrados y abarca tanto las conductas públicas como las privadas con trascendencia pública (quedando excluidas, en principio, aquellas conductas "privadísimas" de las cuales la ciudadanía no tiene conocimiento). Abarca tanto las conductas que tienen lugar en el ejercicio de la función judicial como así también aquellas que se realizan fuera de ella pero que tienen una determinada trascendencia.

No se está ante la presencia de delitos sino de conductas permitidas a nivel privado pero que al tomar repercusión pública adquieren una connotación negativa. Lo que caracteriza principalmente este tipo de comportamientos es que atentan contra la virtud judicial violando, de esta manera, determinados principios éticos.

Se observa la influencia de Aristóteles en la definición expuesta, se interpreta que la mención a "prácticas virtuosas" se construye a partir del concepto de virtud proporcionado por el filósofo griego para quien la virtud es un hábito que busca un punto medio entre extremos y está basado en la prudencia ${ }^{4}$, por ello, se interpreta que con el término prácticas se alude al carácter de repetición que posee un hábito mientras que con el de virtud se toma en igual sentido que para Aristóteles.

\section{Algunas consideraciones genéricas de relevancia:}

\footnotetext{
${ }^{2}$ Ibid.

${ }^{3}$ ANDRUET, Armando (h), "Ámbito de los comportamientos judiciales impropios (l)", Comercio y Justicia, publicado al 19/10/2016, recuperado de https:// comercioyjusticia.info/blog/opinion/ambitos-de-los-comportamientos-judiciales-impropios-i/ (consultado al 28/02/2020).

${ }^{4}$ SZTAJNSZRAJBER, Darío, "Aristóteles", Rizoma / Facultad Libre Virtual, publicado al 25/01/2016, recuperado de https://www.youtube.com/watch?v=QRhPaxS3BPs (consultado al 28/02/2020).
} 
En este punto se esbozan cinco criterios desde los cuales se parte para efectuar el estudio de los CJl, cuatro de ellos expresados de manera afirmativa y uno a modo de varios interrogantes (ello, porque al momento de efectuar el análisis del tema se puede vislumbrar el desprendimiento de una serie de aristas que generan la aparición de mayor cantidad de preguntas que respuestas).

En primer lugar, se debe tener presente que en la figura del juez se pueden distinguir aquellas cuestiones "técnicas" (es decir, lo atinente al "razonamiento judicial" estrictamente), como también las de carácter "ético" (aquellas que se vinculan con determinados principios éticos).

En estas páginas se analizan los comportamientos judiciales a la luz de criterios éticos (es decir, no se pone el énfasis en las cuestiones estrictamente técnicas), pero, no obstante ello, jamas se puede dejar de señalar que al momento de decidir en un caso concreto los magistrados resuelven de acuerdo a ambas categorías: deben tener los conocimientos técnicos necesarios, y, a la vez, ninguno de ellos esta exento de poseer determinados criterios morales, al respecto: "el sistema moral del juez emerge en cada decisión"5.

Este punto es de gran importancia dado que el interrogante que surge tras considerar ambos aspectos presentes en una decisión judicial, puede ser formulado de la siguiente manera: ¿qué sucede en aquellos casos en los cuales las cuestiones técnicas no aparecen tan claramente formuladas debido a que, por ejemplo, no existe una normativa clara para aplicar?, teniendo en cuenta esta pregunta, se puede formular la siguiente: ¿surgen con mayor evidencia los criterios morales de un juez en los casos en los cuales no hay una normativa clara a aplicar?, y, finalmente, tomando en consideración éste último interrogante, da pie a uno nuevo: ¿cómo analizar cuáles son los criterios morales que deberían poseer los magistrados?.

Teniendo en cuenta las preguntas formuladas, se trae a colación el interrogante que plantea Jorge Malem Seña y que da lugar a su trabajo titulado: “ipueden las malas personas ser buenos jueces? ${ }^{6}$ . Aunque existe una crítica atinada al trabajo de dicho autor ya que "En ningún momento queda claro qué define a una persona como "mala" y por qué esta constitución moral afecta el desempeño técnico correcto de jueces que, suponemos, conocen bien el derecho" "7 no obstante, se estima que resulta de utilidad la pregunta que formula Malem Seña dado que sirve como una especie de "disparador" para continuar pensando la temática tratada.

En segundo lugar, resulta de interés mencionar que a medida que las sociedades se pluralizan, en el sentido de que comienzan a aparecer mayores "conflictos" (vistos éstos como una "disputa" entre diversos valores) la figura del juez puede decaer o bien puede fortalecerse, pero, seguramente, se deberán re-pensar determinadas estructuras sociales atento que emergen diversas formas de valoración y los magistrados deben estar atentos y adaptarse a dichos cambios.

En una sociedad plural, en la que es fácilmente distinguible la diversidad valorativa, ¿debe prevalecer algún tipo de moral sobre otra?, ¿cómo saber cual criterio moral debe ser adoptado por el magistrado?.

${ }^{5}$ ANDRUET, Armando (h), "Argumentación Jurídica y la Ética profesional del abogado y el juez", Universidad de San Isidro, publicado al 22/09/2016, recuperado de https://www.youtube.com/watch?v=TE9KAtXZZZ8 (consultado al 18/10/2018) (enlace no disponible al 04/03/2020)..

${ }^{6}$ MALEM SEÑA, Jorge F. “¿Pueden las malas personas ser buenos jueces?”, Universitat Pompeu Fabra- Barcelona, recuperado de http://www.biblioteca.org.ar/ libros/142059.pdf (consultado al 28/02/2020).

LARIGUET, Guillermo. "El aguijón de Aristófanes y la moralidad de los jueces", DOXA, Cuadernos de Filosofía del Derecho, 36 (2013) ISSN: 0214-8676. págs 107126, recuperado de http://ri.conicet.gov.ar/bitstream/handle/11336/14853/CONICET_Digital_Nro.18168.pdf?sequence=1\&isAllowed=y (consultado al 28/02/2020). 
En tercer lugar, hay que tener presente que el valor "justicia" es de difícil definición, incluso para los mismos jueces, este aspecto ya fue advertido por Aristóteles al hacer referencia a lo justo e injusto: "Lo que sucede más comúnmente, es cuando uno de los términos opuestos se toma en muchos sentidos, el otro término, como una derivación necesaria, puede tomarse indistintamente de muchas maneras. Esto ocurre con lo justo y lo injusto. Efectivamente, la justicia y la injusticia pueden entenderse en muchos sentidos, y si, habitualmente, en este caso hallamos la semejanza se debe a que la diferencia de matices no es muy notoria") ${ }^{8}$ (El subrayado me pertenece).

A lo largo de la historia son numerosos los conceptos que pueden encontrarse acerca de lo que se entiende por justicia, incluso en una misma ciudad y en un mismo espacio temporal, un magistrado puede considerar que justicia es algo que para otro no (el ejemplo más claro esta dado en aquellos supuestos en los cuales ante un mismo caso un juez de primera instancia adopta determinado criterio mientras que el de segunda instancia, otro).

Si en los supuestos de resoluciones judiciales es de difícil conocimiento establecer criterios para determinar que una decisión es "más correcta" que otra (y admitiendo que esto es posible), cómo descubrirlo siendo que en general las interpretaciones judiciales deben hacerse dentro de un determinado marco normativo. Si se presentan dificultades en los razonamientos más técnicos, en los casos vinculados a razones morales es un tanto más complejo.

En cuarto lugar, y, luego de tomar postura por aquella visión que considera que los criterios morales de los magistrados se pueden observar de manera más palpable en los supuestos en los cuales no existe una normativa clara, se entiende que los jueces deben ser portadores de determinados criterios morales, al respecto: “(...) existe cierto consenso en relación con las virtudes morales e intelectuales que todo buen juez debería poseer. Estas son la integridad, el coraje, la sobriedad, la sabiduría (teórica y práctica) y la justicia, a las que se opondrían los vicios de corrupción, cobardía, intemperancia, ignorancia e injusticia",

Se alude a que los magistrados deben ser portadores de determinados cualidades morales pues se considera que no todas son igualmente válidas a los fines de la construcción de magistrados virtuosos.

Finalmente, se aportan una serie de interrogantes como punto de partida como así también para pensar posibles fundamentos que sirvan para cuestionar una generalidad de comportamientos impropios que se pongan bajo la mirada de lo ético, tales como: ¿qué es la ética?, ¿por qué la ética es importante al momento de evaluar las decisiones y conductas de los jueces?, ¿qué criterios éticos deben adoptarse cuando se someten a análisis las conductas de los jueces? ¿cómo se construyen esos criterios? ¿quiénes están facultados para la construcción de los mismos? ¿qué incidencia social tiene analizar las conductas de los jueces bajo la lupa de lo ético? ¿existe una ética o moral ejemplar por sobre otros criterios morales? ¿cómo lograr un equilibrio en cuanto a modelos éticos en sociedades plurales?.

${ }^{8}$ ARISTOTELES, "Etica”, Ed Libertador, Buenos Aires, 2009, pág 122.

${ }^{9}$ ASAMAME, Luciana. "Justicia y Empatía: Dificultades y Propuestas", Mendoza, 2016, recuperado de file:///C:/Users/Home\%202013/Downloads/DialnetJusticiaYEmpatia-6182006.pdf (consultado al 28/02/2020). 


\section{La ética judicial}

En este apartado se toma como referencia lo manifestado en la XIII Cumbre Judicial Iberoamericana en cuanto al Código Iberoamericano de Ética Judicial: “(...) La ética judicial incluye los deberes jurídicos que se refieren a las conductas más significativas para la vida social, pero pretende que su cumplimiento responda a una aceptación de los mismos por su valor intrínseco, esto es, basada en razones morales; además, completa esos deberes con otros que pueden parecer menos perentorios, pero que contribuyen a definir la excelencia judicial. ${ }^{10}$ "(....) Esos principios, al procurar modelar el ideal del mejor juez posible, no sólo reclaman ciertas conductas sino que alientan que, tras la reiteración de las mismas, se arraiguen en hábitos beneficiosos, facilitadores de los respectivos comportamientos y fuente de una más sólida confianza ciudadana. ${ }^{11}$

Al respecto, se puede observar:

a) "La ética judicial incluye los deberes jurídicos que se refieren a las conductas más significativas para la vida social", se interpreta que existe una correlación entre las "conductas más significativas" y los "deberes jurídicos", las primeras son el sustento de los segundos, a partir de la existencia de dichas conductas se generan los deberes jurídicos.

No se trata de cualquier tipo de conductas, aquí cabría analizar cuáles son los criterios para determinar esa jerarquía que supone al decir "más significativas", y, a su vez, cuáles son las conductas que quedan comprendidas.

Por otro lado, son conductas significativas a nivel social, por lo que, puede suponerse que implican exteriorización y que no quedarían comprendidas aquellas que no tengan ningún tipo de impacto en otro individuo.

Se observa que se parte de una mirada deontológica al hacer referencia a deberes y no a lo vinculado con el concepto de virtud.

b) "pero pretende que su cumplimiento responda a una aceptación de los mismos por su valor intrínseco, esto es, basada en razones morales, aquí se entiende que para que exista cumplimiento debe haber aceptación del deber y, por otro lado, la aceptación de los mismos depende del valor intrínseco que se les otorgue, para lo cual se tendrán en cuenta razones de carácter moral. Es decir, se interpreta, que se otorga una fundamentación de carácter moral al cumplimiento de los deberes jurídicos.

Ahora bien, a partir de lo manifestado, cabe realizar una distinción: un aspecto es fundamentar el cumplimiento del deber y otra cuestión es el fundamento del deber en sí mismo.

En las líneas citadas se sujeta el "cumplimiento" de los deberes jurídicos a razones de tipo moral (a través de la aceptación), pero, no obstante, queda por zanjar la cuestión del fundamento del deber en sí: ¿puede decirse que también depende de razones de carácter moral?, en aquellos supuestos en donde los deberes no son aceptados por inmorales ¿pierden su carácter de deber o afecta meramente el cumplimiento del mismo?.

${ }^{10}$ XIII CUMBRE JUDICIAL IBEROAMERICANA, “Código Iberoamericano de Ética Judicial” (Punto III: “El Código Modelo como compromiso institucional con la excelencia y como instrumento para fortalecer la legitimación del Poder Judicial"), recuperado de file:///C:/Users/Home\%202013/Downloads/072-2010CE_DA.PDF (consultado al 28/02/2020).

${ }^{11}$ Ibid. (Punto XII: "Los principios éticos como núcleos concentrados de ética judicial”). 
c) "se arraiguen en hábitos beneficiosos, facilitadores de los respectivos comportamientos y fuente de una más sólida confianza ciudadana", aquí puede vislumbrarse la influencia aristotélica dada la utilización del término "hábitos" y a su vez entiende que deben ser "beneficiosos".

Para Aristóteles la virtud es un hábito y si bien no utiliza propiamente el término beneficioso, en cierta forma lo supone: "Es necesario no conformarse, como hecho hasta ahora, con afirmar que la virtud es un hábito o forma de ser, sino que es necesario afirmar también de manera especifica cuál es esa forma de ser.

Partamos por dejar afirmado que la virtud es, en función de la cosa sobre que recae, lo que completa la buena disposición de la cosa y le confirma la realización perfecta de la obra que le incumbe; de manera que la virtud del ojo hace que el ojo sea bueno y que ejecute su función como debe, ya que gracias a la virtud del ojo se ve bien $^{\prime \prime}$. (El resaltado me pertenece).

\section{La ética de la virtud:}

Es importante hacer referencia a la ética de la virtud dado que es un enfoque que amplía los puntos de análisis: "Es un hecho por todos conocido la reactivación que en la década de los 60' del pasado siglo experimentó la ética de la virtud, fundamentalmente en el ámbito de la filosofía anglosajona (...) Tal es, ciertamente, el caso de la filosofí jurídica. Pues los estudios vertidos en su ámbito han estado dominados durante largo tiempo tanto por enfoques de corte deontológico como consecuencialista. Sin embargo, a estos enfoques se ha venido a sumar un tercero: un enfoque que introduce centralmente el concepto de virtud en el dominio de la reflexión jurídica"(...) "Entre las principales temáticas abrazadas por dicha orientación, destaca el estudio de la relación entre la ley y la virtud, y la cuestión de qué tipo de cualidades epistémicos deben poseer los operadores jurídicos para desempeñar de manera excelente su función" "13.

En este sentido, y como se ha explicado, se considera que el análisis desde el concepto de virtud enriquece las preguntas y por lo tanto las respuestas. Ya no es suficiente enfatizar el estudio sólo en las instituciones, también es necesario evaluar qué tipo de magistrados se desea para una sociedad: (...) "un enfoque adecuado para dar cuenta de la moralidad de los jueces o abogados puede ser reconstruido con provecho si utilizamos los aportes de la ética de la virtud y, en vez de hablar sólo de deberes judiciales, hablamos también de virtudes judiciales"14.

La teoría de la virtud es importante porque permite extender el marco de análisis, no es suficiente estudiar la legitimidad de una determinada institución judicial, sino que resulta necesario evaluar qué criterios morales poseen aquellos que la componen.

Además, se dice que se extiende el campo de observación porque ya no alcanza con mirar únicamente el desempeño estrictamente judicial, sino, que será necesario, tener en consideración las conductas desplegadas por los magistrados fuera de la misma ya que se interpreta que para un correcto desempeño se deben poseer determinadas cualidades morales (se poseen en todo momento, se tienen o no).

Como se manifestó en otras líneas, en su decisión el Tribunal Supremo de Puerto Rico consideró que además de una correcta aplicación de la norma se requiere un determinado comportamiento por parte de los jueces. Al respecto, puede señalarse:

\footnotetext{
${ }^{12}$ ARISTOTELES, "Ética”, Ed Libertador, Buenos Aires, 2009, pág 48.

"SAMAME, Luciana. "Justicia y Empatía: Dificultades y Propuestas", Mendoza, 2016, recuperado de file:///C:/Users/Home\%202013/Downloads/DialnetJusticiaYEmpatia-6182006.pdf (consultado al 28/02/2020).

${ }^{14}$ ALARIGUET, Guillermo. "El aguijón de Aristófanes y la moralidad de los jueces", DOXA, Cuadernos de Filosofía del Derecho, 36 (2013) ISSN: 0214-8676. págs 107-
} 126, recuperado de http://ri.conicet.gov.ar/bitstream/handle/11336/14853/CONICET_Digital_Nro.18168.pdf?sequence=1\&isAllowed=y (consultado al 28/02/2020).. 
- Se parte de la hipótesis que una correcta aplicación normativa no alcanza, pero, ahora bien, también debe señalarse que en muchas ocasiones ni siquiera existe una norma a aplicar o bien pueden darse casos dudosos (en los cuales la normativa a aplicar no aparece tan claramente y la interpretación judicial toma mayor énfasis convirtiéndose en la clave para la resolución del conflicto).

Por otra parte, detrás de las interpretaciones judiciales que se dan a conocer en una decisión, se encuentran las cuestiones morales que tienen la particularidad de que en muchas ocasiones las personas pueden no ser conscientes de los criterios morales que poseen.

- Al requerirse un determinado comportamiento por parte de los jueces, la cuestión es descifrar qué tipo de comportamientos, porqué y para qué. (Son interrogantes que exceden estas páginas).

Por último en cuanto a este aspecto es interesante cuestionarse acerca de porqué "reflota" la perspectiva de la ética de la virtud en la década del sesenta y fundamentalmente en la filosofía anglosajona.

Es de importancia continuar en la búsqueda de razones dado que este enfoque, al poner bajo la lupa más específicamente las condiciones que deben tener los magistrados, facilita que salgan a la luz cuestiones que quedan en la mayoría de los casos escondidas por un manto de formalismo, ya que, en muchas ocasiones, pareciera que mientras menos se analicen las razones morales mas "seria" es la decisión. (Tal vez ello, influenciado por las enseñanzas que postulan la separación entre derecho y moral).

No alcanza con aplicar debidamente el derecho, incluso en una aplicación mayormente aceptada socialmente hay cuestiones de indole moral por debajo del razonamiento y es de utilidad analizarlas no solo para aspirar a que los magistrados sean más "integros" sino para entender de una manera más amplia la sociedad de la que se es parte.

\section{La relevancia de los Códigos de Ética:}

En este punto se avanza a partir de lo planteado por Gema Marcilla, cuando manifiesta: "Resulta bastante pacífica la idea de que los códigos éticos son una manifestación de la moral social".

Sin embargo, resulta de interés esbozar una serie de especificaciones, fundamentalmente en lo atinente al concepto de "moral social" dado que pueden ser planteados algunos interrogantes: ¿qué se entiende por "moral social"?, ¿es una o son varias?, ¿hay una moral que "triunfa" sobre otras? ¿la moral que "triunfa" en un contexto es la que termina llamándose "moral social"?.

Se considera que si bien cada código de ética tiene plasmado en su interior deberes éticos para los profesionales y que éstos son creados a partir de determinados criterios morales, resulta de importancia continuar "re-pensando" el concepto de moral social. 
Por otro lado, y en relación a lo abordado en este apartado, es de utilidad hacer mención: "la deontología es la ética profesional concretada en unas normas y códigos de conducta exigibles a los profesionales, aprobadas por el colectivo de profesionales, que enumera una serie de deberes y obligaciones mínimas para todos los profesionales con algunas consecuencias de carácter sancionador" 16 . Aquí puede observarse que en los códigos éticos será posible encontrar determinados deberes para los profesionales, y, a la vez, se establece que serán los mismos profesionales los encargados de establecer cuáles serán. A su vez, también se alude que en algunos supuestos de incumplimiento podrán establecerse consecuencias de tipo sancionador.

Es decir, se mencionan tres aspectos de relevancia: a) los códigos de ética contienen deberes para los profesionales; b) son los profesionales de cada rama los encargados de establecer cuáles serán esos deberes; c) ante el incumplimiento podrá haber consecuencias de tipo sancionador.

En este caso interesa reflexionar de manera más detallada sobre el aspecto que contempla a los profesionales de cada rama como los encargados de establecer cuáles serán los deberes que tendrán que cumplir, y, teniendo en cuenta dicho supuesto se pueden plantear algunas preguntas: ¿es correcto que sean los mismos profesionales los encargados de decidir cuáles serán los deberes que se "autoaplican"?, por otro lado, y suponiendo que esto sea lo correcto, ¿es suficiente con la mera intervención de los profesionales de cada rama?, ¿pueden agregarse actores provenientes de otros sectores para la elaboración del listado de deberes?.

Se hizo hincapié en dicho aspecto porque es el que se considera mayormente vinculado al concepto de "moral social", ya que se interpreta que de acuerdo a los actores intervinientes en la creación de las normas, predominarán determinados criterios morales que terminarán plasmándose, en estos casos, en forma de códigos éticos.

Por otra parte, también interesa reflexionar acerca del tiempo y espacio que funcionan como la atmósfera de la cual emanan las normas éticas, al respecto: "Los códigos deontológicos, nunca emergen en la realidad para ser eternos sino que tienen la sintonía de la inmediatez con un tiempo, lugar y factores sociales en donde ellos han irrumpido. Por lo que, modificadas dichas circunstancias, también muchas veces, debe variar la manera de la realización de la práctica profesional y si ello, no es a su vez correspondido en el instrumento operativo y deontológico profesional, se corre el riesgo que cualquier juzgamiento ético que se pueda hacer, quede naturalmente opacado por una simple cuestión de atemporalidad del canon o porque, la manera de realizarse la práctica en un tiempo y en otro hayan tenido importantes variaciones" ${ }^{\prime 17}$.

Se sigue la línea interpretativa que considera que los códigos éticos están vinculados a un espacio y tiempo determinados ya que son códigos sujetos a cambios y si bien pueden existir algunas similitudes entre algunos de ellos, no son todos estrictamente iguales.

Como se dijo, se comparte la idea de vínculo de los códigos de ética con "la sintonía de la inmediatez con un tiempo, lugar y factores sociales en donde ellos han irrumpido", y, a la vez, se plantea la interrogante: ¿será posible o queda completamente desechada la posibilidad de elaborar determinados criterios éticos que sirvan para la construcción de deberes morales válidos para todo tiempo y lugar?, o incluso, desde un punto de vista menos abarcativo, ¿será posible la elaboración de un código ético aplicable a profesionales de distintas ramas en una misma sociedad?.

${ }^{16}$ Ética en el Ejercicio de la Abogacía- Doctrina y Jurisprudencia del Tribunal de Disciplina de Abogados de la Provincia de Córdoba- Ed Advocatus. Córdoba, 2016, (Capítulo I escrito por Armando Andruet (h)- "Necesaria actualidad del código deontológico para abogados de Córdoba”) pag 25.

${ }^{17}$ Ibid, pág 27. 


\section{Análisis del caso:}

Como se dijo a lo largo de estas páginas, se aborda un CJl llevado a cabo a partir del uso de la red social Facebook, y, al adentrarse específicamente en el caso de la suspensión del juez Eric Colón Colón, se señala que el análisis de dicho comportamiento se efectúa a partir de tres criterios relevantes: en primer lugar, se hace referencia a los argumentos esgrimidos por el juez Colón Colón en su defensa como así también los vertidos por el Tribunal Supremo al desechar las razones proporcionadas por el juez; en segundo lugar, se distingue si se trata de una conducta virtuosa o viciosa desde el punto de vista de la teoría aristotélica como así también tomando en consideración el concepto de CJl, y, finalmente, se utiliza un principio que generalmente es usado para analizar la responsabilidad jurídica, esto es el Principio de Posibilidades Alternativas (PPA) a los fines de evaluar si el juez Colón Colón pudo actuar de otra manera y las consecuencias que ello acarrea desde lo ético.

a) Los principales argumentos del juez Colón Colón para fundamentar su defensa ante la imputación de violación de determinados cánones del Código de Ética de Puerto Rico, pueden resumirse de la siguiente forma:

- Las publicaciones realizadas fueron llevadas a cabo en un ámbito personal y no público.

- Las publicaciones no fueron realizadas ejerciendo su investidura de magistrado.

- Los cánones de ética no tienen ninguna norma referida a las redes sociales.

- No existe una reglamentación precisa respecto al uso de redes sociales para los jueces.

- Las publicaciones vertidas están dentro del marco de su derecho de libre expresión.

Dichos argumentos no fueron suficientes para el Tribunal Supremo quien consideró que en el caso en cuestión por más de que en el Código de Ética no exista una normativa expresa respecto al uso de redes sociales para los magistrados, debe utilizarse el criterio de equiparación a faltas análogas.

Además, dicho cuerpo judicial, también hizo referencia a que el hecho de poseer una red social implica conocer y aceptar que la misma tiene trascendencia pública, por lo que, en este supuesto no funciona en el argumento de lo "privado" esgrimido por el magistrado cuestionado.

Sin embargo, el argumento central de dicho Tribunal para desechar las razones vertidas por el juez es que los cánones éticos se aplican incluso al ámbito de la vida privada de los magistrados dado que la función judicial tiene una gran relevancia a nivel social, no interesa si las conductas fueron desplegadas en ejercicio de la misma o fuera de ella, esto se puede observar en la cita con la que el Tribunal comienza el fallo: "He aqui el dilema: la aplicación correcta de la ley o la norma jurídica no es suficiente para ejercer correctamente la función judicial. Los estilos de trabajos, de comunicación y el comportamiento del juez en y fuera del tribunal son igualmente importantes para la imagen y el logro de la justicia". ${ }^{18}$ (El subrayado me pertenece).

${ }_{18}$ RIVER ROMAN, L. y LOPEZ CINTRON, C. "El temperamento y la función judicial”, 1 Rev, Ley y Foro 4, 2009, recuperado de https://gfr-ph-assets.s3.amazonaws. com/docs/2017/03/31/op.deltribunaljpc.pdf (consultado al 28/02/2020). 
b) Considerando la definición proporcionada por el Dr. Armando Andruet (h) en cuanto a los CJI como así también la teoría de la virtud aristotélica, cabe preguntarse, si se trató de una conducta aislada o si era una forma usual de desempeño de este magistrado, a lo cual se responde que: al tratarse de una serie de publicaciones no se condice con una conducta aislada sino más bien se convierte en una forma usual de expresión del juez.

El hecho de que se trate de una serie de conductas hace posible la vinculación con el concepto de hábito tomado de la teoría aristotélica, dado que se trata de una reiteración de comportamientos (una serie de publicaciones), en esta ocasión de carácter negativo, por lo que, no se puede hablar de una conducta virtuosa, sino, de su opuesto, una conducta viciosa (el término vicio como contrario al de virtud también es tomado del pensamiento de Aristóteles), al respecto: "Consiguientemente, debe anotarse ante todo que la virtud es aquello que debe prepararnos con relación a los dolores y a los placeres de tal modo que nuestra conducta sea la mejor posible, y que el vicio es justamente todo lo opuesto" 19 .

Las publicaciones reiteradas del juez Colón Colón dan origen a un comportamiento vicioso, se trata de una práctica que atenta contra la excelencia a la que deberían aspirar quienes ocupan dichas posiciones.

Por lo que, indagar sobre si se trata de una conducta aislada o recurrente como así también sobre el carácter de la misma (es decir, si es virtuosa o viciosa), tiene importancia si se parte de la idea que el accionar de los magistrados repercute en el grado de valoración que posean los ciudadanos respecto de éstos, de modo que, la ciudadanía podrá ver afectada su confianza hacia dichos funcionarios.

La confianza de los ciudadanos hacia los jueces (y hacia los funcionarios en general) no es una cuestión menor atento que cualquier institución debe tener cierto grado de credibilidad social. Pero, fundamentalmente, evaluar si se esta en presencia de conductas virtuosas o viciosas de los magistrados tiene relevancia a los fines de aproximarse más adecuadamente a la realización del valor justicia.

En este caso en particular la conducta fue llevada a cabo a través de una red social, y, si bien los magistrados pueden hacer uso de los cambios tecnológicos y de comunicación que se van produciendo no los habilita a desplegar conductas impropias a la condición de un juez (sea a través de cualquier medio).

Cuando se habla de magistrados virtuosos se hace referencia a aquellos que lo son dentro y fuera de su función, es decir, los que realizan prácticas prudentes y poseen autocontrol sobre sí mismos: "Se ha entendido al autocontrol como la capacidad que poseen los agentes racionales de tener deseos que se ajusten a aquellos que ellos mismos creen que tendrían si fueran completamente racionales” (...) "¿Cómo podemos saber si una persona es capaz de autocontrol (...)? Sencillamente estudiando su conducta pasada"20.

c) Además, teniendo en cuenta el PPA utilizado generalmente para el análisis de la responsabilidad jurídica y el cual postula: “(...) si una persona puede ser objeto de un reproche moral en virtud de alguna de sus acciones, entonces esto indica que ella podría haber actuado de un modo diferente al que lo hizo" 21 , cabe formularse el interrogante si fue posible que el magistrado obrase de una manera diferente.

\footnotetext{
${ }^{19}$ ARISTOTELES, "Ética”, Ed Libertador, Buenos Aires, 2009, pág 44.

${ }^{20}$ FARREL, Diego Martín. "Entre el Derecho y la Moral. Preguntas con respuestas", Ed Abeledo Perrot, Buenos Aires, 2012, págs 212 y 213.

${ }^{21} \mathrm{lbid}$, pág 216.
} 
A este interrogante se responde de manera afirmativa dado que se entiende que el juez Colón Colón pudo actuar de otra forma: no realizando dichas publicaciones. Ahora bien, que el juez haya podido no realizar dichas publicaciones no significa que no pueda utilizar la red social Facebook, pero, si, al hacerlo debe ser más cuidadoso que otros ciudadanos dado que está sujeto a exposición pública.

EI PPA es de utilidad a los fines de evaluar si el juez pudo haber obrado de una manera diversa ya sea a través de una acción positiva o bien omisiva y en base a ello formular el respectivo reproche, que en este caso es de carácter moral. Pero, no obstante ello, el PPA no puede dar respuesta acerca del carácter correcto o incorrecto de las conductas debidas y las realizadas, para ello, será necesario un análisis más profundo y de tipo filosófico.

Por otra parte, que a determinada conducta u omisión le siga como respuesta la formulación de determinado tipo de reproche, también escapa a este ensayo.

\section{Problemáticas que requieren de respuesta:}

Se parte de la idea que los jueces deben poseer determinadas cualidades morales, el problema está dado en establecer porqué, cuáles son y cómo se formulan.

Las cuestiones atinentes al porqué es de las de más difícil solución o bien el que puede presentar mayor número de opiniones al respecto dado que esta vinculado a la fundamentación estrictamente.

Algunos podrán sostener que no es necesario que los magistrados posean determinadas cualidades morales mientras que, otros si (y dentro de este marco se abre un abanico de posibles fundamentos derivados de distintas corrientes, incluso aquellos que postulan, como el caso del autor Roberto Vigo que las profesiones tienen un origen ético y que las regulaciones de este tipo son anteriores a las jurídicas) ${ }^{22}$.

Cuando se postula que un magistrado debe ser portador de determinadas cualidades morales se lo hace pensando en que ello favorecerá la toma de decisiones por parte de éste, y, que lo llevará, de alguna manera, a optar por la mejor solución en las controversias en las que le toque intervenir (no debe perderse de vista que los jueces, fundamentalmente, toman postura frente a una disputa de intereses).

Todas las personas son titulares de valores morales, en el caso de los jueces el aditamento está dado porque no alcanzaría con que simplemente los posean sino que atento su carácter de funcionarios públicos y la actividad social que despliegan, pareciera que es necesario ir un poco más allá, y, que deban ser portadores de determinados aspectos morales a los fines de contribuir al "orden" social y desempeñar su cargo de la mejor manera posible.

En los hechos es de utilidad plantearse este tipo de interrogantes, ya que, por ejemplo, ¿qué pasaría si un magistrado tiene tendencias a la pedofilia y debe resolver acerca de abusos de menores?, en ese supuesto parece claro que en general se estaría en desacuerdo con que dicho funcionario tomase algún tipo de decisión.

No obstante ello, es factible distinguir distintos tipos de conductas de los jueces, en el caso mencionado aparece con claridad la contradicción pero hay otros en los cuales no se advierte con 
tanta facilidad que el juez deba poseer una determinada cualidad moral por sobre otra. (Ello implica continuar pensando posibles alternativas).

Por otro lado, debe tenerse presente que las sociedades cada vez son mayormente plurales en todo sentido, resulta un desafío lograr un equilibrio entre las posturas morales que pueden encontrarse.

En cuanto a cuáles son los criterios morales que deben poseer los magistrados como así también quiénes son los encargados de formularlos, aparece un tanto más claro. Es posible encontrar algunos criterios en los códigos de ética como así también quienes los elaboran y quienes los aplican.

Resulta un tanto extraño que aparezcan con mayor claridad algunos criterios como así también sujetos encargados de elaborarlos y/o de aplicarlos, y, sin embargo no sea de igual manera con respecto a la fundamentación del porqué la necesidad o no de adoptar determinados criterios morales. (Lo cual lo convierte en otro aspecto de análisis que escapa a este ensayo).

Por último, se considera que es relevante dar razones sobre la justificación de la adopción de determinados modelos de moral por parte de los magistrados ya que ello podría contribuir a aportar argumentos para la fundamentación del sistema judicial en su conjunto, y, tal vez, incluso, hasta una mirada más clara acerca de lo que debe considerarse justo o injusto.

\section{Conclusiones:}

En el caso en cuestión, Colón Colón no debería haber realizado las publicaciones a través de su Facebook dado que se entiende que atentan contra el decoro que deben poseer los magistrados, $y$, a su vez, se ve afectada la imparcialidad de la cual también deben ser portadores esta clase de funcionarios. Incluso, se entiende que realizar publicaciones de este tipo puede dar a conocer la postura que podría llegar a tomar el magistrado con respecto a algunas temáticas.

La conducta del juez Colón Colón es de carácter vicioso tomando en consideración el concepto de CJl como así también los conceptos de virtud y vicio brindados por Aristóteles. Se estima que son acertados los argumentos proporcionados por el Tribunal Supremo y que resultan coherentes con dichas líneas de pensamiento, el concepto de virtud tiene un marco de aplicación amplio: la persona virtuosa lo es dentro y fuera de su función judicial.

Por otra parte, el hecho de que las publicaciones hayan sido realizadas a través de una red social personal es irrelevante ya que se comparte el argumento del Tribunal Supremo en cuanto a que aquella persona que decide ser usuario de una red social (en este caso de Facebook) no puede desconocer que adquiere una cierta trascendencia pública lo que allí se da a conocer.

Además, lo que produce fundamentalmente la red social es otorgar visibilidad y expansión de manera instantánea, funciona como un medio para impartir el mensaje, pero, no debe perderse de vista que en realidad lo central es la conducta del magistrado. No se pone en tela de juicio el carácter personal o no de la vía empleada para impartir el mensaje sino que lo central es la conducta del magistrado.

Por otro lado, las redes sociales sirven para comunicar, ante este tipo de actitudes: ¿qué es lo que 
pretende comunicar el magistrado?, ¿qué tipo de mensaje se deja a los ciudadanos? y ¿por qué debería ser o no tolerado por éstos?.

Resulta de relevancia manifestar que en estas líneas se realizaron observaciones partiendo de conductas públicas y privadas con trascendencia pública, por lo que, en relación con el pensamiento que se ha desarrollado a lo largo de estas páginas puede formularse el siguiente interrogante (fundamentalmente tomando el concepto de virtud de Aristóteles): ¿interesa que la conducta tenga algún tipo de trascendencia pública a los fines de considerar si el magistrado es virtuoso?.

Dicho interrogante se plantea porque se entiende que un magistrado es virtuoso o no lo es, y, por ende, actuará conforme a ello en todos los ámbitos o no podrá hacerlo (no obstante, habrá que ser cuidadosos dado que se estima que una cualidad relevante es la exteriorización de la conducta).

Es importante que los magistrados posean determinadas cualidades morales:

- Contribuye a generar una cierta confianza en los ciudadanos (en este punto cabe preguntarse acerca de si seria posible construir una especie de "moral ejemplar" que no fuese aceptada por la mayoría de los ciudadanos y en ese supuesto qué alternativas podrían formularse).

- $\quad$ Son dichos criterios morales los que emergen con mayor fuerza ante aquellos casos en los cuales no existen normas claras o bien hay ausencia de una normativa específica.

Finalmente, y, para concluir: "Si el derecho es finalmente normativo, moral o legítimo no es una cuestión que sólo dependa del material institucional, sino también de la cuestión de los rasgos de carácter que deberían satisfacer sus funcionarios" (...) Un juez moral o justo, esto es, un juez virtuoso, podría quizá tener la chance de enmendar parcialmente la inmoralidad o la injusticia de la mano de una aplicación virtuosa del derecho. Pero si el derecho es inmoral y los jueces también lo son, prácticamente toda esperanza está perdida". 


\section{Referencias bibliográficas}

ANDRUET, Armando (h). “Argumentación Jurídica y Ética Profesional del Abogado y el Juez”, publicado el 22/09/2016, recuperado de https://www.youtube.com/watch?v=TE9KAtXZZZ8 (consultado al 30/09/2018) ( contenido del enlace no disponible al 04/03/2020).

ANDRUET, Armando (h), “Ámbito de los comportamientos judiciales impropios (I)”, Comercio y Justicia, publicado al 19/10/2016, recuperado de https://comercioyjusticia.info/blog/opinion/ambitos-de-loscomportamientos-judiciales-impropios-i/ (consultado al 28/02/2020).

ANDRUET, Armando (h), “Comportamientos judiciales impropios (II)", recuperado de https://comercioyjusticia. info/blog/opinion/comportamientos-judiciales-impropios-ii/ (consultado al 28/02/2020).

ANDRUET, Armando (h), "Módulo I Fundamentos Generales de la Ética Filosófica y de la Ética Aplicada",

Curso Ética Judicial- Escuela Nacional de la Judicatura, República Dominicana, publicado el 22/01/2014, recuperado de https://www.youtube.com/watch?v=KWxQVc7E8_k (consultado al 28/02/2020).

ARISTOTELES, “Ética”, Ed Libertador, Buenos Aires, 2009.

Ética en el Ejercicio de la Abogacía- Doctrina y Jurisprudencia del Tribunal de Disciplina de Abogados de la Provincia de Córdoba- Ed Advocatus. Córdoba, 2016, (Capítulo I escrito por Armando Andruet (h)- "Necesaria actualidad del código deontológico para abogados de Córdoba").

FARREL, Diego Martín. "Entre el Derecho y la Moral. Preguntas con respuestas", Ed Abeledo Perrot, Buenos Aires, 2012.

LARIGUET, Guillermo. "El aguijón de Aristófanes y la moralidad de los jueces", DOXA, Cuadernos de Filosofía del Derecho, 36 (2013) ISSN: 0214-8676. págs 107-126, recuperado de http://ri.conicet.gov.ar/ bitstream/handle/11336/14853/CONICET_Digital_Nro.18168.pdf?sequence=1\&isAllowed=y (consultado al 28/02/2020).

MALEM SEÑA, Jorge F. “¿Pueden las malas personas ser buenos jueces?”, Universitat Pompeu FabraBarcelona, recuperado de http://www.biblioteca.org.ar/libros/142059.pdf (consultado al 28/02/2020).

MARCILLA CORDOBA, Gema. "A propósito del Código Iberoamericano de Ética Judicial”, recuperado de http://iusfilosofiamundolatino.ua.es/download/Marcilla\%20C\%C3\%B3rdoba,\%20Gema,\%20CIEJ,\%20 Ilatina\%20.pdf (consultado al 28/02/2020).

SAMAME, Luciana. "Justicia y Empatía: Dificultades y Propuestas", Mendoza, 2016, recuperado de file://C:/ Users/Home\%202013/Downloads/Dialnet-JusticiaYEmpatia-6182006.pdf (consultado al 28/02/2020).

SZTAJNSZRAJBER, Darío, “Aristóteles”, Rizoma / Facultad Libre Virtual, publicado al 25/01/2016, recuperado de https://www.youtube.com/watch?v=QRhPaxS3BPs (consultado al 28/02/2020). 
VIGO, Rodolfo "Conferencia sobre la Ética Judicial”, publicado el 29/04/2015, recuperado de https://www. youtube.com/watch? $v=w Z J t W 8 G j R i Q$ (consultado al 28/02/2020).

“Ética Judicial, la Internet y redes sociales", recuperado de https://relacionistas.com/wp-content/uploads/2018/01/ E\%CC\%81tica-Judicial-la-Internet-y-las-redes-sociales.pdf (consultado al 28/02/2020).

\section{Links de casos analizado:}

TSPR, recuperado de https://gfr-ph-assets.s3.amazonaws.com/docs/2017/03/31/op.deltribunaljpc.pdf (consultado al 28/02/2020).

http://www.primerahora.com/noticias/policia-tribunales/nota/

suspendenajuezporcomentariosenredessociales-1215009/ publicado el 31/03/2017 (consultado al 28/02/2020).

https://www.elnuevodia.com/noticias/tribunales/nota/

elsupremosuspendeaunjuezmunicipalporcomentariosenfacebook-2306295/ publicado el 31/03/2017

(consultado al 28/02/2020).

Pamela Güemes Vassallo abogada egresada de la Universidad Nacional de Córdoba (Argentina). https://orcid.org/0000-0003-4294-1274

Pamela pame_g4@hotmail.com 